\title{
Quantitative analysis of muscle and tendon retraction in chronic rotator cuff tears
}

\author{
Meyer, D C ; Farshad, Mazda ; Amacker, Nadja A ; Gerber, Christian ; Wieser, Karl
}

\begin{abstract}
BACKGROUND: Musculotendinous retraction is a limiting factor for repair of long-standing rotator cuff tears. However, it is currently unknown to what extent the muscle and tendon contribute to the degree of total retraction. Further understanding of this may possibly influence the strategy of musculotendinous reconstruction. PURPOSE: To analyze the contribution of muscle and tendon to the process of myotendinous retraction. STUDY DESIGN: Cross-sectional study; Level of evidence, 3. METHODS: Magnetic resonance imaging of 130 shoulders with intact $(\mathrm{n}=20)$ or completely torn supraspinatus tendons was analyzed. Fatty infiltration of the supraspinatus muscle was graded according to Goutallier stages. The degree of retraction of the tendon stump and of the musculotendinous junction was assessed. RESULTS: There were 30 shoulders without evidence of supraspinatus fatty infiltration, 25 with stage 1,23 with stage 2,25 with stage 3 , and 15 with stage 4 changes. The corresponding tear sizes (distance of tendon end from greater tuberosity) were 4, 21, 27, 37, and $41 \mathrm{~mm}$; the distance of the myotendinous junction from the greater tuberosity was $22,33,39,48$, and $48 \mathrm{~mm}$; and the length of the tendons (distance of tendon end to myotendinous junction) was 19, 13, 12, 11, and $8 \mathrm{~mm}$, respectively. In Goutallier stage 3 and above, and in case of a positive tangent sign, the musculotendinous junction was, in $90 \%$ of the cases, retracted to or beyond the glenoid. CONCLUSION: Musculotendinous retraction in chronic rotator cuff tears results mainly from shortening of the muscle fibers but in advanced stages results also from shortening of the tendon tissue itself. The present data demonstrate, for the first time, that the residual tendon stump in a tendon tear does not have the length of the original tendon and is further shortened over time. Therefore, direct anatomic tendon reinsertion will result in lengthening of the supraspinatus muscle greater than what it would have been before the tear.
\end{abstract}

DOI: https://doi.org/10.1177/0363546511429778

Posted at the Zurich Open Repository and Archive, University of Zurich

ZORA URL: https://doi.org/10.5167/uzh-66147

Journal Article

Published Version

Originally published at:

Meyer, D C; Farshad, Mazda; Amacker, Nadja A; Gerber, Christian; Wieser, Karl (2012). Quantitative analysis of muscle and tendon retraction in chronic rotator cuff tears. American Journal of Sports Medicine, 40(3):606-610.

DOI: https://doi.org/10.1177/0363546511429778 


\title{
Quantitative Analysis of Muscle and Tendon Retraction in Chronic Rotator Cuff Tears
}

\author{
Dominik C. Meyer, ${ }^{*}$ MD, Mazda Farshad, ${ }^{*}$ MD, MPH, Nadja A. Amacker, ${ }^{\dagger}$ MD, \\ Christian Gerber, ${ }^{*} \mathrm{MD}, \mathrm{FRCS}$, and and Karl Wieser, ${ }^{* \neq} \mathrm{MD}$ \\ Investigation performed at the Balgrist University Hospital, \\ University of Zurich, Zurich, Switzerland
}

\begin{abstract}
Background: Musculotendinous retraction is a limiting factor for repair of long-standing rotator cuff tears. However, it is currently unknown to what extent the muscle and tendon contribute to the degree of total retraction. Further understanding of this may possibly influence the strategy of musculotendinous reconstruction.
\end{abstract}

Purpose: To analyze the contribution of muscle and tendon to the process of myotendinous retraction.

Study Design: Cross-sectional study; Level of evidence, 3.

Methods: Magnetic resonance imaging of 130 shoulders with intact $(n=20)$ or completely torn supraspinatus tendons was analyzed. Fatty infiltration of the supraspinatus muscle was graded according to Goutallier stages. The degree of retraction of the tendon stump and of the musculotendinous junction was assessed.

Results: There were 30 shoulders without evidence of supraspinatus fatty infiltration, 25 with stage 1, 23 with stage 2, 25 with stage 3 , and 15 with stage 4 changes. The corresponding tear sizes (distance of tendon end from greater tuberosity) were 4 , $21,27,37$, and $41 \mathrm{~mm}$; the distance of the myotendinous junction from the greater tuberosity was $22,33,39,48$, and $48 \mathrm{~mm}$; and the length of the tendons (distance of tendon end to myotendinous junction) was 19, 13, 12, 11, and 8 mm, respectively. In Goutallier stage 3 and above, and in case of a positive tangent sign, the musculotendinous junction was, in $90 \%$ of the cases, retracted to or beyond the glenoid.

Conclusion: Musculotendinous retraction in chronic rotator cuff tears results mainly from shortening of the muscle fibers but in advanced stages results also from shortening of the tendon tissue itself. The present data demonstrate, for the first time, that the residual tendon stump in a tendon tear does not have the length of the original tendon and is further shortened over time. Therefore, direct anatomic tendon reinsertion will result in lengthening of the supraspinatus muscle greater than what it would have been before the tear.

Keywords: chronic rotator cuff tear; myotendinous retraction; supraspinatus tendon length; quantitative MRI analysis

The standard surgical treatment of symptomatic rotator cuff tears that fail nonoperative management is the direct repair of the tendon stump to its anatomic insertion on the tuberosities. However, open and arthroscopic repairs of chronic large tears have a substantial structural failure rate. $^{7,22}$ Currently, musculotendinous retractions associated with increased muscular stiffness, reduced muscular work capacity, atrophy, and fatty infiltration are considered to be the main factors for the difficulties in

\footnotetext{
${ }^{\ddagger}$ Address correspondence to Karl Wieser, MD, Orthopedic Department, Balgrist University Hospital, Forchstrasse 340, CH-8008 Zurich, Switzerland (e-mail: karl.wieser@balgrist.ch).

${ }^{*}$ Orthopedic Department, Balgrist University Hospital, University of Zurich, Zurich, Switzerland.

${ }^{\dagger}$ Department of Radiology, University Hospital of Zurich, Zurich, Switzerland.

The authors declared that they have no conflicts of interest in the authorship and publication of this contribution.
}

The American Journal of Sports Medicine, Vol. XX, No. X DOI: $10.1177 / 0363546511429778$

(C) 2011 The Author(s) overcoming tendon retraction and obtaining structural healing. $9,10,13,14,22$ Strategies to deal with this retraction have included thorough musculotendinous release, repair with postoperative rehabilitation with the arm in abduction, medialization of the tendon insertion onto the humeral head, ${ }^{15}$ release of the entire muscle origin, ${ }^{3,12}$ or employing the use of a structural graft to bridge the gap between the anatomic tendon insertion and remaining tendon. ${ }^{1,2,18}$ To determine which of the aforementioned would be most beneficial, it would be necessary to quantitatively understand the individual contribution of the muscle and the tendon to this pathophysiological process. However, quantitative data on the retraction of the rotator cuff musculature, as a consequence of rotator cuff tear, are lacking in the current literature.

We hypothesized that, in chronic large rotator cuff tears, it is not only the muscle that retracts but also the tendon. Therefore, the aim of this present study was to compare the length of both the muscle and tendon of the supraspinatus in patients with intact and with torn rotator cuff tendons. 


\section{MATERIALS AND METHODS}

\section{Materials}

Our prospective patient database, which includes patients from 2001 to 2010, was searched for patients with either a full-thickness supraspinatus or a multitendinous rotator cuff tear. One hundred ten shoulders with standardized preoperative magnetic resonance arthrography scans undergoing either arthroscopic rotator cuff repair or reverse total shoulder arthroplasty for irreparable cuff tear with pseudoparalysis of the shoulder were included. Another 20 shoulders from patients with anterior-inferior shoulder instability without any clinical or radiological signs of cuff disease, as determined by magnetic resonance arthrography, were included as a control group, resulting in a total number of 130 shoulders from 127 patients. After first inspection of the available 130 magnetic resonance imaging (MRI) scans, 12 shoulders had to be excluded because of insufficient imaging quality, incomplete set of images or operative data, or interposition of the supraspinatus tendon into the joint space between the humeral head and glenoid. This resulted in a definitive study group of 118 shoulders in 116 patients (39 left and 80 right shoulders). There were 50 women and 66 men, and the mean age was 53 years (range, 16-81 years).

\section{Methods}

All surgical reports were reviewed for the exact description of tendons involved in the rotator cuff tear. In our institution, all patients underwent magnetic resonance arthrography on a 1.5-T MRI unit (Symphony, Siemens, Munich, Germany) after injection of approximately $12 \mathrm{~mL}$ (range, 10-14 mL) of gadopentetate dimeglumine (solution with a concentration of $2 \mathrm{mmol} / \mathrm{L}$, Magnevist, Bayer Schering Pharma, Berlin, Germany). The shoulder was placed in a dedicated receive-only shoulder coil with the arm in a neutral position and the thumb pointing vertically.

Magnetic resonance arthrography protocols included T1-weighted spin echo images in the coronal oblique plane with fat saturation (792/20; section thickness, $3 \mathrm{~mm}$; field of view, $160 \times 160 \mathrm{~mm}$; matrix size, $265 \times 512$ ), in the transverse plane (500/30; section thickness, $3 \mathrm{~mm}$; field of view, $160 \times 160 \mathrm{~mm}$; matrix size, $256 \times 512$ ), and in the sagittal oblique plane $(500 / 30$; section thickness, $4 \mathrm{~mm}$; field of view, $160 \times 160 \mathrm{~mm}$; matrix size, $256 \times$ $512)$. T2-weighted fast spin echo images (3000/20; section thickness, $4 \mathrm{~mm}$; field of view, $160 \times 160 \mathrm{~mm}$; matrix size, $256 \times 512)$ and intermediate-weighted fast spin echo images (2350/20; section thickness, $4 \mathrm{~mm}$; field of view, $160 \times 160 \mathrm{~mm}$; matrix size, $256 \times 512$ ) were obtained in the coronal oblique plane with fat saturation. Patients with technically comparable magnetic resonance arthrographies performed in other institutions were included into the study group. The MRI data were stored on a picture archiving and communication system (PACS) workstation, and the provider's image analysis software was used for review and measurement of images.

Degree of Fatty Infiltration. The degree of fatty infiltration of all rotator cuff muscles was assessed on MRI

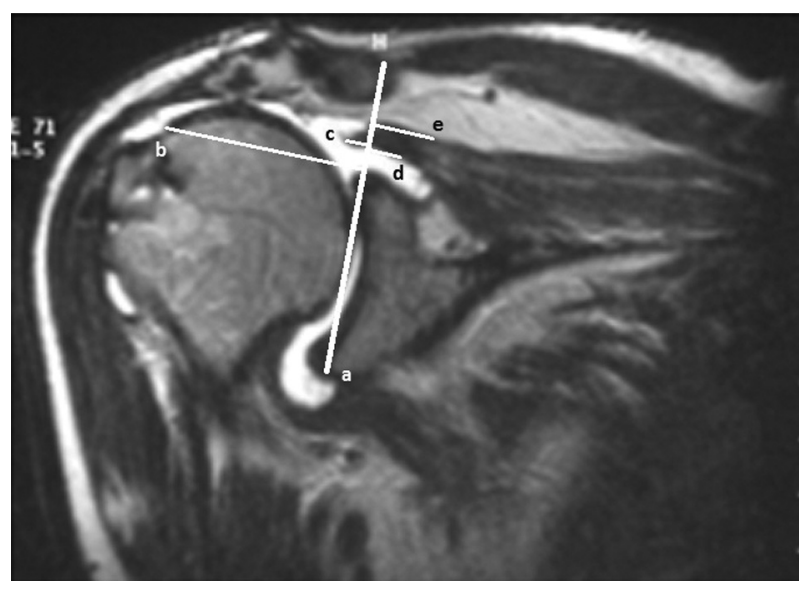

Figure 1. Magnetic resonance arthrography image showing a right shoulder with a transmural rupture and significant pronounced retraction of the supraspinatus tendon with illustration of the measurement method. Line "a" is drawn as a connection line from the supraglenoidal and infraglenoidal tubercles and is functioning as a starting point of all perpendicular measurements. Line "b" measures the distance from this starting point to the footprint (lateral edge of the articular surface). Line "c" measures the retracted tendon end. Lines " $d$ " and "e" are measuring the myotendinous junction on the deep (scapular, articular) and superficial (fascial, bursal) sides, respectively. Tear size was calculated by subtracting distance "c" from "b." Muscle retraction was calculated by subtracting distance " d"'/" e" from " $b$. ." Tendon length was finally calculated by subtracting tear size from muscle retraction.

according to the methods of Fuchs et al, ${ }^{6}$ a grading system that is based on the Goutallier classification, ${ }^{10}$ which has been established for computed tomographic scans. Grading was on the most lateral parasagittal T1-weighted image on which the scapular spine was in contact with the scapular body. The stages identified were as follows: stage 0 , normal muscle; stage 1 , some fatty streaks; stage 2 , manifest fatty infiltration but less fat than muscle; stage 3 , as much fat as muscle; stage 4, more fat than muscle.

Atrophy of the Supraspinatus. Atrophy of the supraspinatus muscle was assessed using the tangent $\operatorname{sign}^{21}$ : A line was drawn from the top of the coracoid process to the top of the spine of the scapula. If the superior border of the muscle was below the tangent line, it was considered positive; if the muscle belly intersected with the line, the tangent sign was negative.

Retraction of the Supraspinatus. On a T2-weighted oblique coronal section through the center of the supraspinatus tendon, the tear size (distance from the lateral edge of the humeral articular surface to the tendon end) and the superficial (fascial, bursal) and deep (scapular, articular) myotendinous junctions (muscle retraction) were measured (Figure 1). The myotendinous junction was defined as the most lateral point where inserting muscle fibers on the central tendon could be detected. Transition areas with markedly increased signal intensity compared with normal cuff tissue were included in the tear size. Adjacent 
TABLE 1

Tear Size, ${ }^{a}$ Muscle Retraction, ${ }^{b}$ and Calculated Tendon Length (Bursal and Articular Sides) of the Supraspinatus Muscle in Different Goutallier Stages

\begin{tabular}{|c|c|c|c|c|c|}
\hline & $\begin{array}{c}\text { Goutallier Stage } \\
0(\mathrm{n}=30)\end{array}$ & $\begin{array}{c}\text { Goutallier Stage } \\
1(\mathrm{n}=25)\end{array}$ & $\begin{array}{c}\text { Goutallier Stage } \\
2(\mathrm{n}=23)\end{array}$ & $\begin{array}{c}\text { Goutallier Stage } \\
3(\mathrm{n}=25)\end{array}$ & $\begin{array}{c}\text { Goutallier Stage } \\
4(\mathrm{n}=15)\end{array}$ \\
\hline Tear size, mm & $3.5 \pm 11.0$ & $20.7 \pm 10.0$ & $26.9 \pm 10.3$ & $36.7 \pm 8.3$ & $40.6 \pm 6.9$ \\
\hline Muscle retraction, $\mathrm{mm}$ & $22.1 \pm 9.2$ & $33.3 \pm 9.6$ & $39.3 \pm 10.9$ & $47.5 \pm 9.6$ & $48.1 \pm 7.2$ \\
\hline Tendon length bursal, mm & $13.1 \pm 7.1$ & $12.9 \pm 6.1$ & $13.7 \pm 6.7$ & $12.4 \pm 6.2$ & $10.0 \pm 3.8$ \\
\hline Tendon length articular, mm & $18.6 \pm 6.1$ & $12.6 \pm 5.2$ & $12.4 \pm 5.9$ & $10.7 \pm 6.0$ & $7.5 \pm 4.8$ \\
\hline
\end{tabular}

${ }^{a}$ Distance from greater tuberosity to tendon end.

${ }^{b}$ Distance from greater tuberosity to myotendinous junction.

images were used to clarify the anatomic relationships and to identify the intramuscular course of the central tendon. By subtracting the value of the measured tendon end to the myotendinous junction, the length of the supraspinatus tendon stump was calculated. These calculations were made for both the bursal and the articular sides of the myotendinous junction (tendon length bursal side and tendon length articular side, respectively).

All included studies were randomly interpreted by 3 independent observers: 2 orthopaedic surgeons with 4 to 5 years' training in orthopaedic and particularly shoulder surgery and 1 radiologist with 2 years of special training in musculoskeletal radiology.

\section{Statistical Analysis}

Statistical analysis was performed with a commercial software package (Prism, version 4 for Macintosh, GraphPad Software Inc, La Jolla, California) under the guidance of a professional statistician. Data were tested for normal distribution before either parametric or nonparametric tests were employed. For categorical data, 1-way analysis of variance (ANOVA) for repeated measurements was used to measure interobserver reliability among the 3 observers. The $R^{2}$ values range from 0 to 1 , with values close to 1 indicating high reliability of measurement. Correlations between mean values of different stages of fatty infiltration, muscle atrophy, and tear size/muscle retraction or tendon length were calculated using Spearman rank correlation or Pearson correlation for nonnormally distributed or normally distributed data, respectively. Level of significance was set at $P<.05$.

\section{RESULTS}

Evaluation of the surgical protocols of the study group showed that 18 shoulders had an intact rotator cuff. All lesions of the rotator cuff were specified as full-thickness tears. In 15 shoulders, only the supraspinatus tendon was affected. The supraspinatus tear extended into the infraspinatus tendon in 27 cases and into the subscapularis tendon in 12 cases, and all 3 tendons were involved in 46 cases.

\section{Fatty Infiltration and Atrophy of Rotator Cuff Muscles}

There was fatty supraspinatus muscle infiltration of Goutallier stage 0 in 30 shoulders (mean age, 34 years; range, 16-57 years), stage 1 in 25 shoulders (mean age, 52 years; range, 37-70 years), stage 2 in 23 shoulders (mean age, 56 years; range, 43-73 years), stage 3 in 25 shoulders (mean age, 63 years; range, 39-79 years), and stage 4 in 15 shoulders (mean age, 70 years; range, 56-81 years). A positive tangent sign, ${ }^{21}$ indicating severe atrophy of the supraspinatus, was found in 52 of the 118 shoulders.

The interobserver correlations among the 3 observers for assessment of the tangent sign and the Goutallier stages of the supraspinatus were $0.8\left(R^{2} ; P<.0001\right)$ and $0.88\left(R^{2} ; P<.0001\right)$, respectively (Appendix, available in the online version of this article at http://ajs.sagepub. com/supplemental/). The $R^{2}$ values for the Goutallier stages of the remaining rotator cuff muscles were as follows: infraspinatus superior part, 0.91 ; infraspinatus inferior part, 0.86; subscapularis superior part, 0.84; and subscapularis inferior part, 0.86 .

\section{Retraction of the Supraspinatus and Tendon Length}

Interobserver correlations for measuring the tear size and the musculotendinous junction are shown in Table 1. As expected, the tear size increased proportionally to the advancement of the Goutallier stages, with $4 \mathrm{~mm}$ in stage $0,21 \mathrm{~mm}$ in stage $1,27 \mathrm{~mm}$ in stage $2,37 \mathrm{~mm}$ in stage 3 , and $41 \mathrm{~mm}$ in stage 4 . The measured muscle retraction (distance from greater tuberosity to muscle) increased as well, with $22 \mathrm{~mm}$ in stage $0,33 \mathrm{~mm}$ in stage $1,39 \mathrm{~mm}$ in stage 2 , and $48 \mathrm{~mm}$ in stages 3 and 4 . The stump of the supraspinatus tendon (tendon length) showed a mean length of $13 \mathrm{~mm}$ and $19 \mathrm{~mm}$ (bursal and articular sides, respectively) in Goutallier stage 0 and decreased with increasing degrees of supraspinatus fatty degeneration, leading to a tendon stump of $10 \mathrm{~mm}$ on the bursal side and $8 \mathrm{~mm}$ on the articular side in Goutallier stage 4 .

A positive tangent sign, indicating severe atrophy of the supraspinatus muscle, was also associated not only with increased retraction of the corresponding tendon $(36 \mathrm{~mm}$ vs $14 \mathrm{~mm}$ with negative tangent sign) but also with a shortened tendon stump when measuring the myotendinous junction on the articular side. The tendon measured $15 \mathrm{~mm}$ in negative and $11 \mathrm{~mm}$ in positive tangent signs. In cases of a positive tangent sign, the myotendinous junction was retracted to or medial to the level of the glenoid in $90 \%$ of patients (mean, $7.8 \mathrm{~mm}$ medial).

The same findings were seen when comparing the Goutallier stages to the myotendinous junction. In Goutallier stage 3 , the myotendinous junction was retracted behind the level of the glenoid in over $90 \%$ of the cases. And no single case of Goutallier stage 4 showed a myotendinous 
junction that was not retracted behind the glenoid. The relationship between supraspinatus quality (fatty muscle infiltration, Goutallier stages) and tear size, muscle retraction (articular side), and calculated length of the tendon stump (articular side) is depicted in Figure 2.

\section{DISCUSSION}

Musculotendinous retraction is generally accepted as the most important pathophysiological consequence of chronic tendon tears and is a major limiting factor for successful surgical repair of the tendon end to the anatomic insertion site. Along with the retraction, the muscle undergoes atrophy and fatty degeneration ${ }^{10}$ with the functional consequences of decreased muscle elasticity, ${ }^{13}$ strength, and therefore loss of work capacity and range of motion of the joint. ${ }^{16}$ Although structural failure after large or massive rotator cuff repairs does not necessarily imply a poor clinical outcome, ${ }^{7,14}$ failure has been shown to be associated with inferior restoration of strength, lower shoulder scores, and more rapid progression of degenerative joint changes than structural healing of repairs. ${ }^{8,22}$ Hence, successful repair of a rotator cuff tear is desirable.

Intuitively, it would appear reasonable to assume that with repair of the torn tendon end to its original insertion site, anatomic restoration of the musculotendinous unit should be possible. However, it has been shown that not only the muscle fibers but also the tendon tissue itself has the potential for shortening. ${ }^{5,17}$ Therefore, it is important to know whether and to what degree the tendon itself has shortened in addition to the extent of muscle retraction. It appears clear that reinsertion of a shortened tendon end will require an excessive lengthening of the corresponding muscle, possibly resulting in undue stretching of this muscle.

Our results document that musculotendinous retraction occurs mostly through shortening of the muscle fibers up to Goutallier stage 3 (as much fat in the muscle as muscle substance). However, we observed that in Goutallier stage 0 to stage $1,6 \mathrm{~mm}$ of tendon length is lost on the articular side. This tendon shortening then remains remarkably unaltered until stages 2 to 3 . Between stages 3 and 4, however, muscle length remains almost unaltered, but the tendon length shortens by another 3 to $4 \mathrm{~mm}$.

From these results, we therefore conclude that tendon and muscle shortening are not synchronous after tendon tear. This is reflected in the remarkably weak correlation between tendon length and muscle retraction $\left(R^{2}=0.049\right)$. The mechanism by which the tendon shortens as the rotator cuff tear progresses cannot conclusively be answered within this present study. However, it appears likely that the initial shortening corresponds to remaining tendon substance that is left on the greater tuberosity after the initial tear, whereas substance loss in the later stages of musculotendinous retraction may be because of either active shortening of the tendon substance, analogous to Wolff law, ${ }^{20}$ which has been observed previously in a sheep model for rotator cuff tears,${ }^{17}$ or reabsorption of the tendon itself.

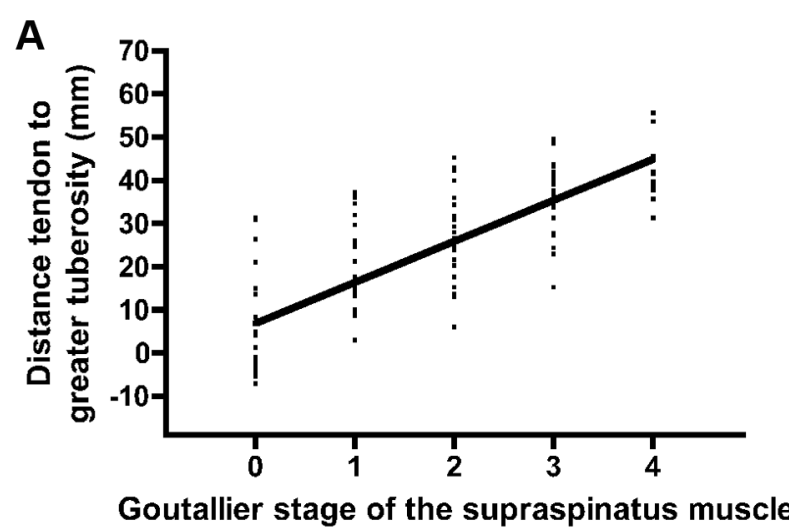

B

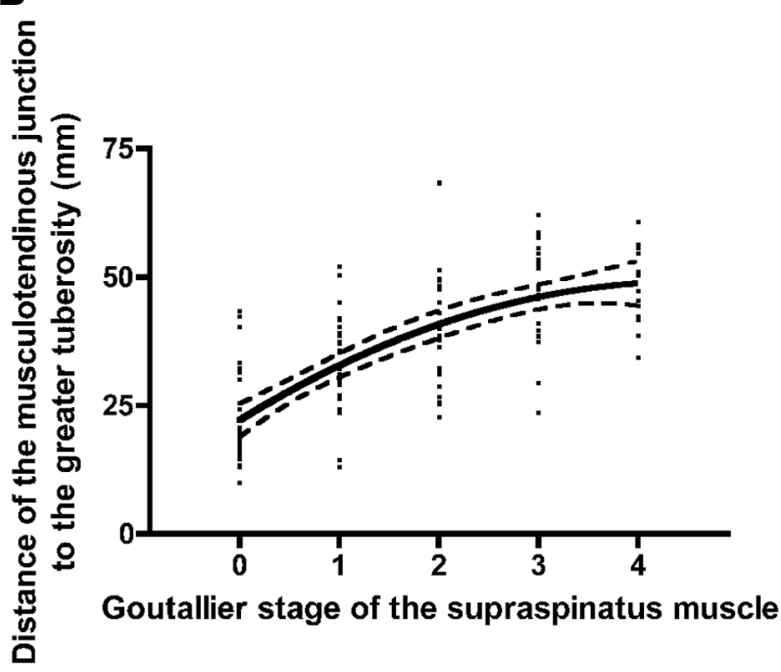

C

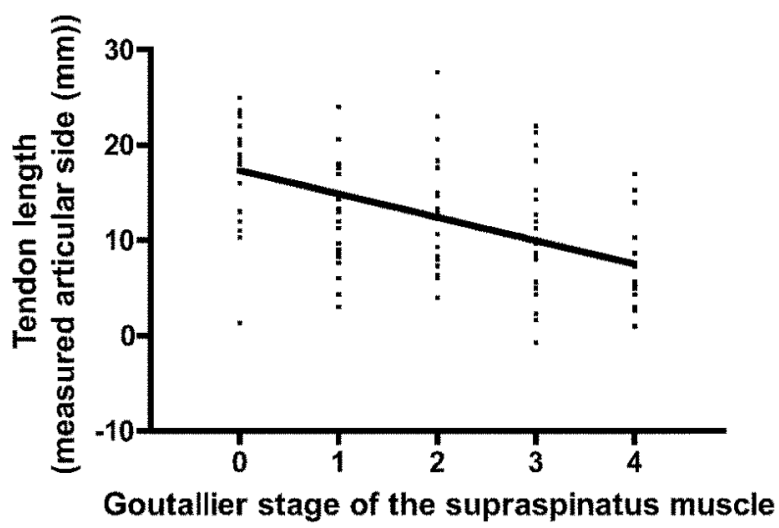

Figure 2. Correlation of supraspinatus muscle quality (Goutallier stages) and retraction of the free tendon end from the greater tuberosity. (A) A proportional enlargement of the tear size with increasing Goutallier stages. The measured muscle retraction (articular side) (B) increased as well, with a flattening of the curve in Goutallier stages 3 and 4 . The calculated length of the tendon stump (articular side) (C) decreased over the increasing Goutallier stages. Overall, $1 \mathrm{~cm}$ of tendon length got lost until Goutallier stage 4. 
Potential limitations exist in the interpretation of these results. The measurement of the muscle insertion can be difficult and sometimes ambiguous to differentiate. Nevertheless, we found a favorable interobserver agreement not only in investigating the Goutallier qualitative grading system and muscle atrophy (tangent sign) but also in measuring the degree of tendon and muscle retraction. Furthermore, from our assessment of the MRI scans, it is often difficult to differentiate if a ruptured and shortened tendon is retracted into the muscle or just absorbed at its distal end. Apposition of scar tissue at the site of rupture may also influence these findings. In addition, this study consists of analysis of a 3-dimensional structure on a 2-dimensional magnetic resonance image, which may contribute to the differences in values between the bursal and articular sides. In future studies, 3-dimensional analysis of the problem may help to better understand the described findings. Measurements were performed using magnetic resonance arthrography, which is the most sensitive $(91.7 \%)$ and specific $(96.5 \%)$ technique for diagnosing both full- and partial-thickness rotator cuff tears. ${ }^{4}$ However, the influence on the myotendinous retraction because of an increased intra-articular pressure after instillation of the contrast agent is currently not known.

On the basis of the assumption that the size of the tear and degree of fatty infiltration give a relative indication about the elapsed time since the tear, we believe that the biomechanical consequences of these radiological measurements help to understand difficulties in the repair of long-standing cuff tears. For example, in Goutallier stage 2, where repair is generally considered possible ${ }^{10,11}$ the tendon end is retracted by approximately $27 \mathrm{~mm}$ from the greater tuberosity, and the muscle has a resting length that is reduced by approximately $20 \mathrm{~mm}$. When an attempt is made to surgically reinsert the tendon to the original footprint, the muscle has to be stretched by $27 \mathrm{~mm}$, as the tendon tissue is practically inelastic. From previous studies, it has been shown that the muscle fiber length of an intact supraspinatus muscle is approximately $57 \pm 0.1 \mathrm{~mm}$ and accordingly less $(33 \pm 6.5 \mathrm{~mm})$ in a retracted supraspinatus. ${ }^{19}$ Therefore, this implies that to reattach the torn tendon to its anatomic insertion site, the necessary muscle fiber lengthening is approximately $100 \%$, which is physiologically not possible without disruption and subsequent scarring of the muscle fibers.

To our knowledge, this is the first quantitative report on muscle retraction after supraspinatus tendon rupture. This study clearly shows that the retraction after a tendon tear is predominantly because of shortening of the muscle belly. Moreover, we demonstrate that in tears with a low degree of fatty muscle infiltration, the tendon is already shortened to a substantial degree, suggesting that overreduction and lateral transposition of the tendon over the greater tuberosity may be unphysiological. Therefore, the remaining length of the muscle may be the better indicator for later function as compared with the gap between tendon end and insertion site. However, if the retraction is indeed mainly attributed to tendon loss, augmentation of the tendon may be a rational consideration.

\section{REFERENCES}

1. Barber FA, Herbert MA, Coons DA. Tendon augmentation grafts: biomechanical failure loads and failure patterns. Arthroscopy. 2006;22:534-538.

2. Bright RW, Green WT. Freeze-dried fascia lata allografts: a review of 47 cases. J Pediatr Orthop. 1981;1(1):13-22.

3. Debeyre J, Patie D, Elmelik E. Repair of ruptures of the rotator cuff of the shoulder. J Bone Joint Surg Br. 1965;47:36-42.

4. De Jesus JO, Parker L, Frangos AJ, Nazarian LV. Accuracy of MRI, MR arthrography, and ultrasound in the diagnosis of rotator cuf tears: a meta-analysis. AJR Am J Roentgenol. 2009;192(6):1701-1707.

5. Farshad M, Gerber C, Snedeker JG, Frauenfelder T, Meyer DC. Structure of retracted tendons after staged repair following continuous traction [published online ahead of print February 12, 2011]. Knee Surg Sports Traumatol Arthrosc. doi:10.1007/s00167-011-1430-3.

6. Fuchs B, Weishaupt D, Zanetti M, Hodler J, Gerber C. Fatty degeneration of the muscles of the rotator cuff: assessment by computed tomography versus magnetic resonance imaging. J Shoulder Elbow Surg. 1999;8(6):599-605.

7. Galatz LM, Ball CM, Teefey SA, Middleton WD, Yamaguchi K. The outcome and repair integrity of completely arthroscopically repaired large and massive rotator cuff tears. I Bone Joint Surg Am. 2004;86(2):219-224.

8. Gerber C, Fuchs B, Hodler J. The results of repair of massive tears of the rotator cuff. J Bone Joint Surg Am. 2000;82(4):505-515.

9. Gerber C, Schneeberger AG, Hoppeler H, Meyer DC. Correlation of atrophy and fatty infiltration on strength and integrity of rotator cuff repairs: study in thirteen patients. J Shoulder Elbow Surg. 2007;16(6):691-696.

10. Goutallier D, Postel JM, Bernageau J, Lavau L, Voisin MC. Fatty muscle degeneration in cuff ruptures: pre- and postoperative evaluation by CT scan. Clin Orthop Relat Res. 1994;304:78-83.

11. Goutallier D, Postel JM, Lavau L, Bernageau J. Impact of fatty degeneration of the supraspinatus and infraspinatus muscles on the prognosis of surgical repair of the rotator cuff. Rev Chir Orthop Reparatrice Appar Mot. 1999;85(7):668-676.

12. Ha'Eri GB, Wiley AM. "Supraspinatus slide" for rotator cuff repair. Int Orthop. 1980;4(3):231-234.

13. Hersche O, Gerber C. Passive tension in the supraspinatus musculotendinous unit after long-standing rupture of its tendon: a preliminary report. J Shoulder Elbow Surg. 1998;7(4):393-396.

14. Jost B, Pfirrmann CW, Gerber C. Clinical outcome after structural failure of rotator cuff repairs. J Bone Joint Surg Am. 2000;82(3):304-314.

15. McLaughlin HL. Repair of major cuff ruptures. Surg Clin North Am. 1963;43:1535-1540.

16. Meyer DC, Gerber C, Von Rechenberg B, Wirth SH, Farshad M. Amplitude and strength of muscle contraction are reduced in experimental tears of the rotator cuff. Am J Sports Med. 2011;39(7):1456-1461.

17. Meyer DC, Lajtai G, von Rechenberg B, Pfirrmann CW, Gerber C. Tendon retracts more than muscle in experimental chronic tears of the rotator cuff. J Bone Joint Surg Br. 2006;88(11):1533-1538.

18. Neviaser JS, Neviaser RJ, Neviaser TJ. The repair of chronic massive ruptures of the rotator cuff of the shoulder by use of a freeze-dried rotator cuff. J Bone Joint Surg Am. 1978;60(5):681-684.

19. Tomioka $\mathrm{T}$, Minagawa $\mathrm{H}$, Kijima $\mathrm{H}$, et al. Sarcomere length of torn rotator cuff muscle. J Shoulder Elbow Surg. 2009;18(6):955-959.

20. Wolff J. Book title: Das Gesetz der Transformation der Knochen (Reprints Medizinhistorischer Schriften, Ausgabe 1982; Berlin, Hirschwald). Chapter title: Die Bedeutung der inneren Architektur der Knochen. Schattauer, Stuttgart 1991.

21. Zanetti M, Gerber C, Hodler J. Quantitative assessment of the muscles of the rotator cuff with magnetic resonance imaging. Invest Radiol. 1998;33(3):163-170.

22. Zumstein MA, Jost B, Hempel J, Hodler J, Gerber C. The clinical and structural long-term results of open repair of massive tears of the rotator cuff. J Bone Joint Surg Am. 2008;90(11):2423-2431. 